light sources to enhance the visibility of bruising should be undertaken with caution.

\section{CAN TRENDS IN CORONIAL POST-MORTEM EXAMINATIONS BE DETERMINED FROM THE NCIS DATABASE?}

Kelly Olds $^{1}$, Michael Rodriguez ${ }^{2}$, Neil Langlois ${ }^{1}$

${ }^{1}$ Forensic Science South Australia, Adelaide, and The University of Adelaide, School of Medical Sciences, Adelaide, SA, and ${ }^{2}$ Prince of Wales Hospital, Randwick, NSW, Australia

There have been changes to Coronial Acts in some States to allow limited examinations or promote the minimum examination necessary to determine a cause of death. Has this impacted on Coronial autopsy numbers and the proportion of partial or external only examinations? An answer to this question was sought using the National Coronial Information Service (NCIS) database. However, this service does not code post-mortem examinations as external, partial or full. An automated method to extract the data using key phrases was devised and tested. However, it was only possible to apply this data extract technique to two States. The trends over ten years will be shown and the data discussed.

\section{KAWASAKI DISEASE: A REVIEW OF 2 CASES OF SUDDEN DEATH}

Sarah Parsons, Matthew Lynch

Victorian Institute of Forensic Medicine and Monash

University, Vic, Australia

Kawasaki disease is a febrile, usually self-limited illness of childhood, which commonly starts with an arteritis. We will present two cases of Kawasaki disease leading to sudden death; one in the acute phase and one 22 years after the initial illness. The first case was a 5-month-old infant who presented to a tertiary hospital with a febrile illness, conjunctivitis and erythema of the skin of the hands. Kawasaki disease was considered in the list of differentials, however as he responded to antibiotic treatments he was discharged home. At autopsy the deceased was found to have a haemopericardium with a ruptured coronary artery aneurysm. He had widespread arteritis.

The second case was that of a 22-year-old man who collapsed whilst playing football. He had been diagnosed at 6 months of age with Kawasaki disease complicated by left and right coronary artery aneurysms.

Autopsy findings (gross and histological) and PM CT images will be presented in these cases along with a review of current guidelines into diagnosis.

\section{SUDDEN CARDIAC DEATH WHILST PLAYING AUSTRALIAN RULES FOOTBALL: A RETROSPECTIVE 14 YEAR REVIEW}

Sarah Parsons, Matthew Lynch

Victorian Institute of Forensic Medicine and Monash

University, Vic, Australia
Australian Rules football (AFL) is a sport unique to Australia. It is played and followed widely in most states of Australia but particularly in Victoria. The death of a player during a professional or recreational game of AFL is usually widely reported and affects not only the family but the whole club, often raising questions about whether more can be done to ensure that sudden cardiac deaths don't occur in these settings. Pre-participation screening is often discussed as a way to avoid sudden cardiac deaths at least at a semi-professional level.

A good understanding of the autopsy findings in young men who die during particular sport will help to add to the discussion on whether testing will help to decrease the incidence of these deaths occurring.

This paper will examine 14 cases where sudden cardiac death has occurred whilst playing AFL over a 14 year period in the state of Victoria in young males. This cohort demonstrates a wide variety of cardiac findings both expected and unexpected.

There will be a discussion on the variety of autopsy findings which range from possible anatomic causes of death, to genetic finding and other causes (such as commotio cordis).

The contribution of anatomical or genetic findings alone as the cause of death will also be explored.

Given the wide variety of causes of death in this cohort it is probable that pre-participation screening may have been able to detect a small number of cases but most would have gone undetected.

\section{VEHICLE ASSISTED LIGATURE STRANGULATION}

Bianca Phillips, Nathan Milne

Forensic and Scientific Services, Health Support Queensland, Queensland Department of Health, Qld, Australia

This is a case report of an unemployed young adult male with a history of substance abuse and recent domestic dispute that committed suicide by vehicle assisted ligature strangulation (VALS). VALS is a rare method of suicide in which a motor vehicle is used to apply pressure to the neck by a ligature, which is fixed to a structure outside of the vehicle. There are only a handful of cases previously documented in the literature. The findings in these cases are varied and show one of three patterns of injury: complete decapitation, lethal injury to the internal structures of the neck or sustained pressure to the neck. This case showed a combination of findings with both significant injury to the internal structures of the neck and features of sustained pressure to the neck. Review of the literature showed that some of the variation in pattern of injury seen could be attributed to the intactness of the ligature. It is likely that other factors also play a role including the force applied by the motor vehicle and the characteristics of the ligature used such as material and width. Further case reports are required to further our understanding of this rare mechanism of suicide.

\section{FATAL ARACHNOPHOBIA}

Bianca Phillips, Dianne Little

Gold Coast University Hospital, Queensland Department of Health, Qld, Australia 